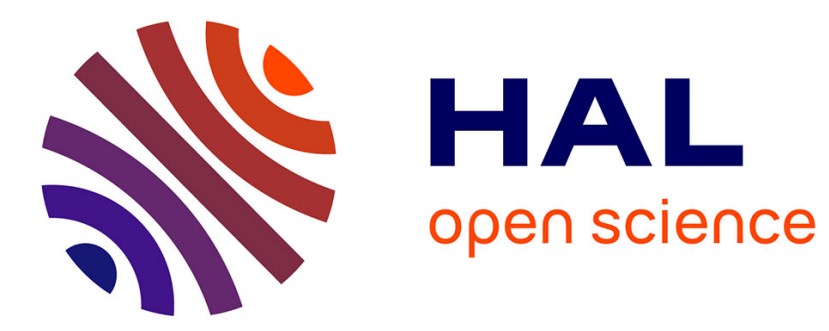

\title{
Peeling-angle dependence of the stick-slip instability during adhesive tape peeling
}

Marie-Julie Dalbe, Stéphane Santucci, Loïc Vanel, Pierre-Philippe Cortet

\section{To cite this version:}

Marie-Julie Dalbe, Stéphane Santucci, Loïc Vanel, Pierre-Philippe Cortet. Peeling-angle dependence of the stick-slip instability during adhesive tape peeling. Soft Matter, 2014, 10 (48), pp.9637-9643. 10.1039/C4SM01840K . hal-01727329

\section{HAL Id: hal-01727329 \\ https://univ-lyon1.hal.science/hal-01727329}

Submitted on 12 Mar 2018

HAL is a multi-disciplinary open access archive for the deposit and dissemination of scientific research documents, whether they are published or not. The documents may come from teaching and research institutions in France or abroad, or from public or private research centers.
L'archive ouverte pluridisciplinaire HAL, est destinée au dépôt et à la diffusion de documents scientifiques de niveau recherche, publiés ou non, émanant des établissements d'enseignement et de recherche français ou étrangers, des laboratoires publics ou privés. 


\title{
Peeling-angle dependence of the stick-slip instability during adhesive tape peeling
}

\author{
Marie-Julie Dalbe ${ }^{a b}$ Stéphane Santucci, ${ }^{a}$ Loïc Vanel $^{b}$ and Pierre-Philippe Cortet,${ }^{c}$
}

\author{
Received Xth $X X X X X X X X X X$ 20XX, Accepted $X$ th $X X X X X X X X X$ 20XX \\ First published on the web $X$ th $X X X X X X X X X X 200 X$
}

DOI: $10.1039 / \mathrm{b} 000000 \mathrm{x}$

The influence of peeling angle on the dynamics observed during the stick-slip peeling of an adhesive tape has been investigated. This study relies on a new experimental setup for peeling at constant driving velocity while keeping constant the peeling angle and peeled tape length. The thresholds of the instability are shown to be associated with a subcritical bifurcation and a bistability of the system. The velocity onset of the instability is moreover revealed to strongly depend on the peeling angle. This could be the consequence of a peeling angle dependance of either the fracture energy of the adhesive-substrate joint or the effective stiffness at play between the peeling front and the point at which the peeling is enforced. The shape of the peeling front velocity fluctuations is finally shown to progressively change from typical stick-slip relaxation oscillations to nearly sinusoidal oscillations as the peeling angle is increased. We suggest that this transition might be controlled by inertial effects possibly associated to the propagation of the peeling force fluctuations through elongation waves in the peeled tape.

\section{Introduction}

In standard fracture mechanics, crack growth is usually described by a velocity-dependent fracture energy, accounting for the rate dependence of the energy cost of elementary dissipative rupture processes close to the fracture tip $^{1}$. Then, the condition for a crack to propagate at a given velocity is that the amount of mechanical energy released by a unit area of crack growth provides the corresponding fracture energy ${ }^{2}$. When fracture energy becomes a decreasing function of crack velocity, i.e. it costs less energy for the crack to grow faster, a dynamical instability often occurs. In that case, the crack velocity starts to oscillate as well as the energy release rate. Such oscillations are a common feature of crack propagation and can be observed for very different ranges of mean crack velocity depending on the considered material ${ }^{3,4}$. The stick-slip oscillations observed during the peeling of adhesive films is a very-well known example of such dynamic rupture instability $^{5-8}$.

The unstable "stick-slip" dynamics of adhesive film

${ }^{a}$ Laboratoire de Physique de l'ENS Lyon, CNRS and Université de Lyon, France

${ }^{b}$ Institut Lumière Matière, UMR5306 Université Lyon 1CNRS, Université de Lyon, France.

${ }^{c}$ Laboratoire FAST, CNRS, Univ. Paris Sud, France.

M.-J. Dalbe, E-mail: mariejulie.dalbe@ens-lyon.fr; S. Santucci, E-mail: stephane.santucci@ens-lyon.fr; L. Vanel, E-mail: loic.vanel@univ-lyon1.fr; P.-P.Cortet, E-mail: ppcortet@fast.upsud.fr. peeling is admitted to be the consequence of a decrease of the fracture energy $\Gamma\left(v_{p}\right)$ of the substrate-adhesive joint within a specific range of fracture velocity $v_{p}$ combined with the compliance between the location where the peeling velocity $V$ is imposed and the peeling fracture front $^{5-12}$. This decrease has been proposed to proceed from the viscoelasticity of the adhesive material coupled to the effects of material confinement and large deformations ${ }^{13-17}$. Peeling an adhesive tape from a freely rotating roller is a standard configuration which has received much experimental and theoretical attention ${ }^{7,8,12,18-24}$. However, in some circumstances, the stick-slip peeling may become intermittent when pendulum-like oscillations of the roller develop as a result of the interplay between the peeling force and the roller inertia ${ }^{23}$. This intermittent behavior has been attributed to the possibility of an intrinsic dependance of the instability with the peeling angle which, in these experiments, oscillates quasi-statically as a consequence of the slow unsteady roller dynamics ${ }^{23}$.

In order to study properly the influence of the peeling angle on the stick-slip instability, about which very few experimental results exist ${ }^{6}$, we have developed an innovative experimental setup where the adhesive tape end is pulled at a controlled velocity from a plane substrate which is translated at the same velocity. Unlike usual peeling geometries allowing to control the peeling angle, we can also keep fixed the length of the peeled tape, which controls the elastic compliance of the peeling system and is an important control parameter of the instability. Furthermore, 


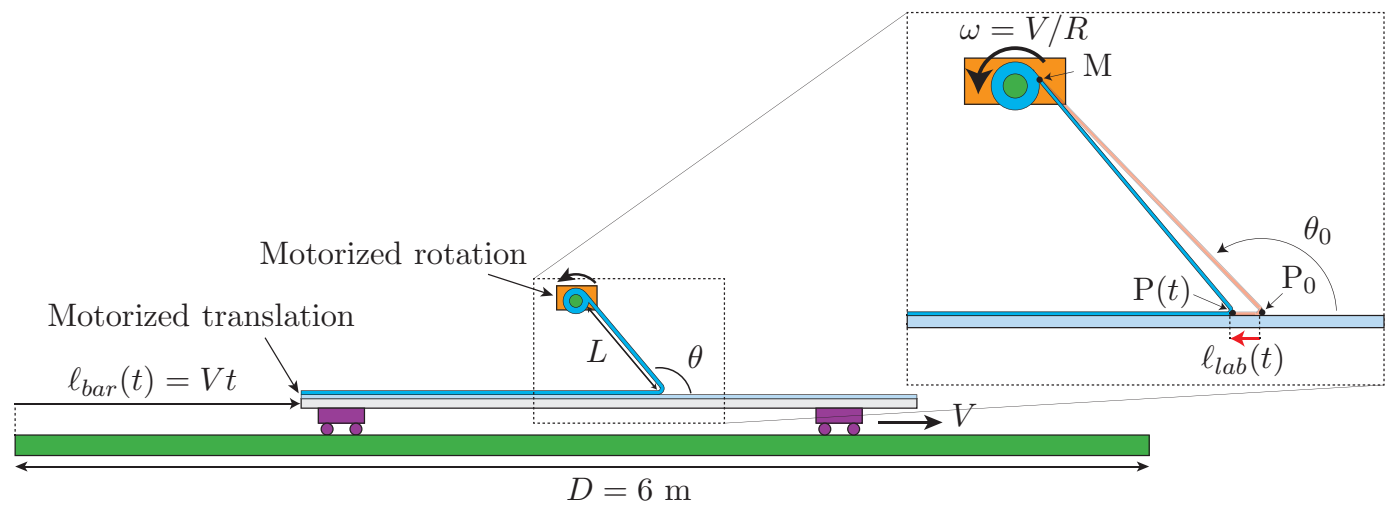

Figure 1: Scheme of the peeling experiment at controlled velocity $V$, angle $\theta$ and peeled tape length $L$. The translation velocity $V$ of the rigid bar and the winding velocity $R \omega$ are slaved electronically to each other. $\ell_{b a r}$ is the position of the rigid bar and $\ell_{l a b}(t)=\overline{P_{0} P}(t)$ the position of the peeling front in the laboratory frame. $M$ is the location of the point where the winding of the peeled tape proceeds, $P_{0}$ the average location of the peeling point and $P(t)$ its location as a function of time $t . \theta_{0}$ is the average peeling angle. We denote $L=\left|\mathbf{M P}_{\mathbf{0}}\right|$ the average peeled tape length.

in contrast with the roller geometry, the inertia of the substrate (which becomes effectively infinite) will not be anymore a parameter of the peeling problem. This new setup, associated to a high speed imaging of the fracture dynamics, has allowed us to quantify for the first time, at fixed peeled tape length, the dependance of the instability velocity thresholds and amplitude with peeling angle.

\section{Experimental methods}

The experimental setup (Fig. 1) consists in a $3 \mathrm{~m}$ long, $45 \mathrm{~mm}$ wide rigid bar which can be translated at a controlled velocity $V$ up to $4.5 \mathrm{~m} \mathrm{~s}^{-1}$ thanks to a CC servomotor. The bar is covered with a layer of adhesive tape which constitutes the substrate of a second layer of the same adhesive. The adhesive tape, 3M Scotch ${ }^{\circledR} 600$ (as in refs. ${ }^{12,22-24}$ ), is made of a polyolefin blend backing ( $38 \mu \mathrm{m}$ thick, $19 \mathrm{~mm}$ wide, Young modulus $E=1.26 \mathrm{GPa}$ ) coated with a $20 \mu \mathrm{m}$ layer of a synthetic acrylic adhesive. The experiments have been performed at a temperature of $22.3 \pm 0.9^{\circ} \mathrm{C}$ and a relative humidity of $43 \pm 9 \%$.

During an experiment, the top layer adhesive tape is peeled from its substrate thanks to a second servo-motor which is winding the peeled tape on a cylinder of radius $R$ at a rotation rate $\omega$ (Fig. 1). The rotation rate $\omega$ is slaved electronically to the velocity of the rigid bar translation, i.e. $\omega(t) \propto V(t)$, such that $R \omega(t) \simeq V(t)$ even during acceleration and deceleration transients of the experiment. Whatever the target velocity $V$ of the experiment -between 0.03 and $4.5 \mathrm{~m} \mathrm{~s}^{-1}$ - the two motors are able to accelerate and decelerate enough strongly so that a stationary regime at velocity $V$ can be observed over at least $1 \mathrm{~m}$ of peeling. When the imposed velocity $V$ does not fall in the stick-slip unstable range, the peeling front velocity $v_{p}(t)$ is constant and kinematically set to $V$ by this system. In this situation, the coupled translation and winding motions actually impose to the peeling point to remain fixed in the laboratory frame, setting consequently the peeling angle $\theta(t)$ and the peeled tape length $|\mathbf{M P}|$ to constant values (see Fig. 1).

It is worth to point two experimental subtleties that need to be managed carefully for the experimental setup to work properly. First, if one sets the winding velocity $R \omega$ and the translation velocity $V$ to the exact same value, a slow drift of the peeling point in the laboratory frame is observed during the stationary phase of the experiment. This drift is due to the fact the tape is peeled from the substrate in an unstreched state whereas it is wound in a stretched state. Second, during the peeling, the radius $R(t)$ of the winding cylinder -initially of $39.5 \mathrm{~mm}$ - increases of $0.6 \%$ per meter of peeled tape due to the thickness of the tape being wound, which drives an acceleration of the winding with respect to the translation. In practice, we compensate these two effects simultaneously by setting the target winding rotation rate $\omega$ to a value slightly faster (of a few $0.1 \%)$ than the target translation velocity $V$. With this method, we succeed to limit the slow drift of the peeling point in the laboratory frame during the stationary stage of peeling to values corresponding to drifts of the peeling angle lower than $1^{\circ}$ and drifts of the peeled tape length lower than $3 \%$.

The real value of our setup is that when the stick-slip instability is present, the instantaneous fluctuations of the peeling angle and of the peeled tape length still remain 
small: throughout all the experiments presented in this paper, they are respectively ranging from $0.1^{\circ}$ to $2^{\circ}$ and from $0.1 \%$ to $5 \%$. At the same time, the peeling front velocity oscillates strongly with amplitudes larger or much larger than $V$. Note however that the peeling front velocity follows precisely the imposed velocity $V$ once averaged over timescales larger than the stick-slip instability period.

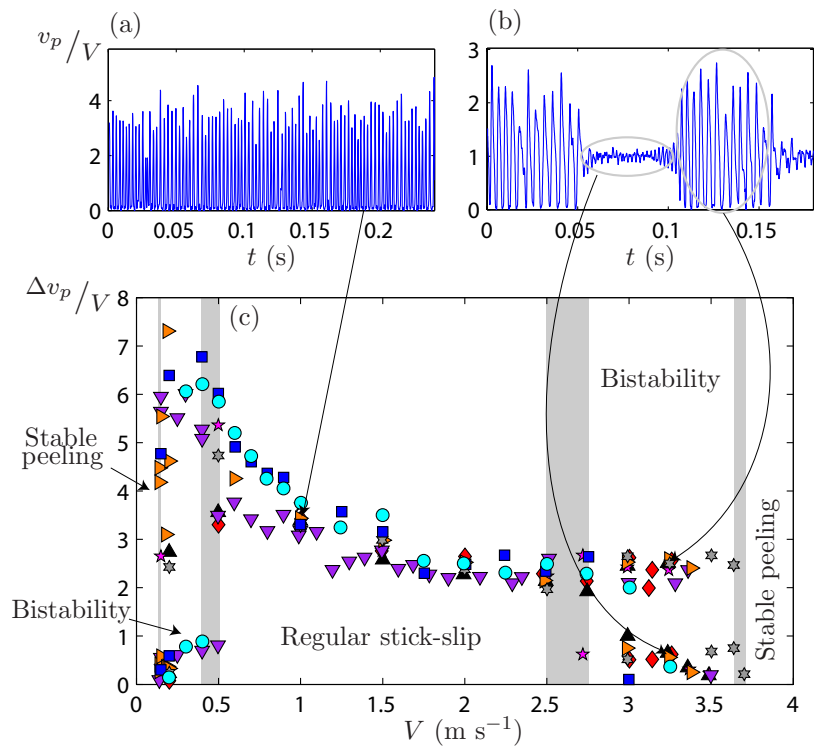

Figure 2: Portions of the measured time series $v_{p}(t) / V$ for typical experiments at $\theta=90^{\circ}$ : (a) $L=45 \mathrm{~cm}, V=$ $1 \mathrm{~m} \mathrm{~s}^{-1}$, regular stick-slip; (b) $L=69 \mathrm{~cm}, V=3.25 \mathrm{~m} \mathrm{~s}^{-1}$, bistable peeling. (c) Statistical average $\Delta v_{p} / V$ of the amplitude of fracture velocity fluctuations $\delta v_{p}(t) / V$ as a function of $V$ for $\theta=90^{\circ}$ and various peeled tape lengths $L$ ( $\mathbf{\nabla}: L=25 \mathrm{~cm} ; \boldsymbol{\Delta}: L=34 \mathrm{~cm} ; \star: L=45 \mathrm{~cm} ; \nabla$ : $L=54 \mathrm{~cm}$; : $L=69 \mathrm{~cm} ; *: L=81 \mathrm{~cm} ; \mathbf{\square}: L=100 \mathrm{~cm}$; -: $L=134 \mathrm{~cm})$. The standard deviation of $\delta v_{p}(t) / V$ over all velocity fluctuations in one experiment is typically of the order of the symbol size.

The peeling dynamics is imaged up to 20000 frames/s (Photron Fastcam APX RS). The corresponding images of $896 \times 512$ to $384 \times 224$ pixels have a resolution in the range 40 to $80 \mu \mathrm{m} /$ pixel. From the recorded image time series, we detect the location of the peeled tape in the laboratory frame at a small distance of $0.7 \pm 0.1 \mathrm{~mm}$ from the substrate. This measurement provides an estimate for the position $\ell_{l a b}(t)$ of the fracture front in the laboratory frame (Fig. 1) and is used to compute the velocity $d \ell_{l a b} / d t$ with typical errors of $\pm 1 \%$. We also measure the instantaneous velocity of the substrate, $d \ell_{\text {bar }} / d t$, with a typical relative precision of $\pm 0.4 \%$. We finally compute the fracture velocity relative to the substrate $v_{p}(t)=d \ell_{l a b} / d t+d \ell_{\text {bar }} / d t$.

\section{A subcritical instability}

In Fig. 2(a), we show a sequence of the peeling fracture velocity time series $v_{p}(t) / V$ for a typical experiment for which stick-slip instability is observed, consisting in high frequency alternation between slow and fast phases of peeling. When the peeling is unstable, we typically observe from a few dozen (for $\theta=30^{\circ}$ ) to a few hundred (for large $\theta$ ) stick-slip cycles during the complete stationary regime of peeling in one experiment. Over this statistical ensemble of stick-slip oscillations in a given experiment, we always observe a very stable period of stick-slip from cycle to cycle, which illustrates that the stick-slip instability has reached a "stationary state".

In the following, we focus on the characterization of the instability velocity amplitude as a function of the peeling control parameters $V, \theta$ and $L$. To do so, we start by defining, from the fracture velocity time series, the instantaneous amplitude of the peeling instability as the velocity contrast, $\delta v_{p}(t)=\max \left(v_{p}(t), t \in[t-T / 2, t+T / 2]\right)-$ $\min \left(v_{p}(t), t \in[t-T / 2, t+T / 2]\right)$, between the maximum and minimum values of the fracture velocity $v_{p}(t)$ over a sliding time interval $T$ of the order of the typical stick-slip cycles duration. Practically, we also compute this quantity when stick-slip instability is not present, in which case $\delta v_{p}$ measures the amplitude of the fracture velocity fluctuations due to spatial heterogeneities in adhesion or to the fluctuations of the velocity enforced by the motors.

In Fig. 2(c), we report the average of the amplitude of fracture velocity fluctuations $\Delta v_{p} / V=\left\langle\delta v_{p}(t)\right\rangle / V$ as a function of the driving velocity $V$ for several experiments performed with a peeling angle $\theta=90^{\circ}$ and various peeled tape lengths $L$. For imposed velocities $V$ below $V_{\text {onset }}=0.135 \pm 0.005 \mathrm{~m} \mathrm{~s}^{-1}$ and above $V_{\text {disp }}=$ $3.7 \pm 0.04 \mathrm{~m} \mathrm{~s}^{-1}$, the peeling dynamics is stable with limited velocity fluctuations $\left|\Delta v_{p} / V\right|<0.2 \pm 0.01$. For peeling velocities up to $V=0.45 \pm 0.05 \mathrm{~m} \mathrm{~s}^{-1}$, a change in the fracture dynamics occurs with the appearance of intermittencies between phases of a noisy but rather stable peeling and phases of well-developed stick-slip instability (Fig. 2(b)). In this bistable regime, the probability distribution of $\delta v_{p}(t)$ has two bumps with a minimum around $1.5 \mathrm{~V}$. We analyze separately events with $\delta v_{p}<1.5 \mathrm{~V}$ corresponding to noisy stationary peeling and events with $\delta v_{p}>1.5 \mathrm{~V}$ corresponding to stick-slip peeling. This data processing leads to two average values $\Delta v_{p}$ in Fig. 2(c) which are characteristic of the coexisting two types of peeling dynamics. For peeling velocities $V$ larger than $0.45 \pm 0.05 \mathrm{~m} \mathrm{~s}^{-1}$, bistability is no longer present and the fully developed stick-slip regime of peeling is the unique stable state of the system. A typical time series of $v_{p}(t) / V$ in the pure stick-slip regime is provided in Fig. 2(a). For 
$2.63 \pm 0.13<V<V_{\text {disp }}=3.7 \pm 0.04 \mathrm{~m} \mathrm{~s}^{-1}$, the peeling dynamics is bistable again, and can be characterized by two average values of $\delta v_{p} / V$ for a given peeling velocity. The bistability of the peeling dynamics at the appearance and disappearance thresholds suggests that the stick-slip instability onsets as a function of the imposed velocity $V$ are associated to subcritical bifurcations. This result constitutes a very valuable information that excludes theoretical models predicting supercritical bifurcations (at least for the considered adhesive tape $)^{8}$.

\section{Impact of peeling angle}

We study now how the features of the instability reported in Fig. 2 for a peeling angle of $\theta=90^{\circ}$ depend on the two control parameters that are the peeled tape length and the peeling angle. In Fig. 2(c), we see that the different instability thresholds and the value of the instability amplitude do not significantly depend on the length of the peeled tape $L$, at least over the limited range of $L$ investigated here, from 25 to $134 \mathrm{~cm}$. This invariance moreover remains a robust feature when varying $\theta$. On the contrary, the instability thresholds depend strongly on the peeling angle as can be seen in Figs. 3 and 4 . Fig. 3 presents a state diagram in the $(V, \theta)$-space showing the domains where the peeling is stable, bistable or fully unstable. We find that the velocity range over which the adhesive peeling is dynamically unstable tends to increase of at least an order of magnitude as the peeling angle decreases. This is observed for the two frontiers between stable and bistable, as well as between bistable and stick-slip regimes. For $\theta \leqslant 60^{\circ}$, the limitations of the experimental setup in peeling velocity $V$ did not allow us to reach neither the low velocity stable domain, nor the high velocity bistable and stable domains. For $\theta$ increasing above $120^{\circ}$, the velocity thresholds seem to saturate to constant values. It is important to highlight here that, to our knowledge, only Aubrey et $a l .{ }^{6}$ had previously reported evidence for a dependence of the instability with the peeling angle thanks to the observation of the disappearance of the instability when $\theta$ is increased at a specific imposed velocity. They however did not performed a systematic study of the instability as a function of the control parameters and the peeled tape length was varying during a tensile test. The marked dependence of the stick-slip instability with the peeling angle that we report here is consequently an important feature that has however not been considered by current theories of adhesive peeling instability. This point is discussed in more details in the final paragraphs of the paper.

Figure 4 finally shows the dependance of the velocity

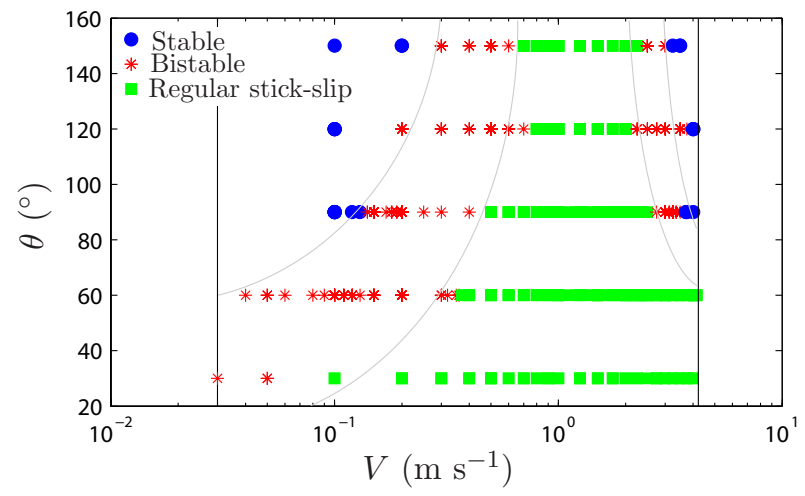

Figure 3: Diagram of the peeling regime in $(V, \theta)$-space. Each marker corresponds to one experiment. The vertical lines show the experimental limits of our setup. Continuous gray lines are guides for the eyes.

amplitude $\Delta v_{p}$ of the dynamical instability with peeling angle $\theta$ and driving velocity $V$. Each data point corresponds here to an average of $\delta v_{p}(t)$ over experiments for which the peeling is fully unstable or on time intervals over which stick-slip is observed for bistable experiments. Taking advantage of the fact that the instability amplitude $\Delta v_{p}(V, \theta, L)$ does not depend significantly on the peeled tape length $L$, data for a fixed velocity $V$ and peeling angle $\theta$ are averaged over $L$. For a given peeling velocity $V$, the instability amplitude decreases with the peeling angle $\theta$, initially rapidly for small angles but more slowly as $\theta$ increases. The instability amplitude finally seems to saturate to a low limit value for $\theta \geqslant 120^{\circ}$. The dependance of the instability amplitude $\Delta v_{p}$ with the peeling velocity $V$ actually changes drastically with $\theta$. For $\theta=30^{\circ}$, the instability amplitude is nearly constant with the peeling velocity $V$ whereas, as $\theta$ increases up to $150^{\circ}, \Delta v_{p}(V)$ tends towards linearity with $V$. The strong difference in behavior between the stick-slip velocity amplitude at small and large peeling angle is an intriguing result.

\section{Discussion}

\subsection{Effect of peeling angle on the instabil- ity thresholds}

To get some insight on the reported effects of peeling angle on the stick-slip instability, we start from the dynamical equations derived originally by Barquins et al. ${ }^{7}$. In stationary peeling condition, the adhesive peeling dynamics is described by the balance equation $G=\Gamma\left(v_{p}\right)$ between a fracture energy $\Gamma\left(v_{p}\right)$, which accounts for the energy dissipated near the fracture front, and the strain energy release 


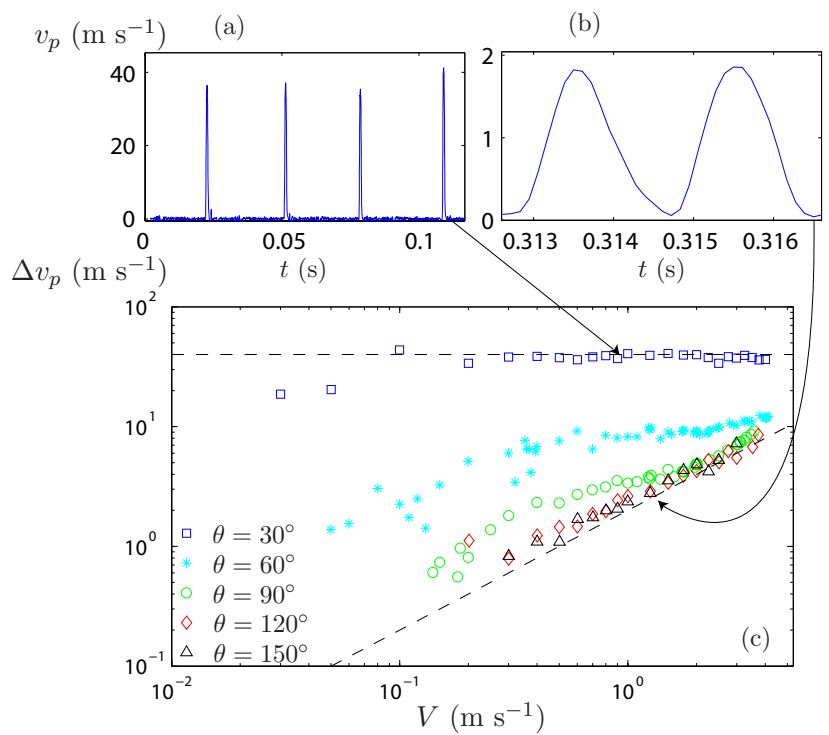

Figure 4: Short portions of the measured time series of the instantaneous peeling velocity for (a) $V=0.9 \mathrm{~m} \mathrm{~s}^{-1}$, $\theta=30^{\circ}, L=0.51 \mathrm{~m}$ and (b) $V=0.9 \mathrm{~m} \mathrm{~s}^{-1}, \theta=150^{\circ}$, $L=0.54 \mathrm{~m}$. (c) Instability amplitude $\Delta v_{p}$ as a function of peeling velocity $V$ for different peeling angles $\theta$. Each marker represents data averaged over different peeled tape length $L$. The lower dashline is $\Delta v_{p}=2 V$ and the upper one $\Delta v_{p}=40 \mathrm{~m} \mathrm{~s}^{-1}$.

rate $G$ which corresponds to the release of mechanical energy, both per unit surface of fracture growth. The strain energy release rate is ${ }^{25}$

$$
G=\frac{F}{b}(1-\cos \theta)+\frac{F^{2}}{2 b^{2} e E} \simeq \frac{F}{b}(1-\cos \theta),
$$

where $F$ is the force transmitted to the fracture along the tape, $b$ the tape width, $e$ its thickness and $E$ its Young modulus. Neglecting the second term in Eq. (1), corresponding to the elastic energy stored in the tape, is a very good approximation for most adhesive tapes and peeling geometries. In our case, this term goes from 2 to 3 orders of magnitude smaller than the first term when $\theta$ increases from $30^{\circ}$ to $150^{\circ}$.

In order to estimate the force $F$ in the peeled tape, we need to generalize for any peeling angle the relationship between the tape elongation $u$ and the peeling velocity $v_{p}$ which was originally expressed as $\mathrm{d} u / \mathrm{d} t=V-v_{p}$ in ${ }^{7}$, but, as we show below, is valid for $\theta=90^{\circ}$ only. To that aim, we introduce the following notations (see Fig. 1): $M$ is the location of the point where the winding of the peeled tape proceeds, $P(t)$ the location of the peeling point as a function of time $t$ and $P_{0}$ its average location during a stick-slip cycle (for stationary peeling, $P(t)=P_{0}$ ). In the reference frame of the laboratory, the peeling location $P(t)$ tends to move at a velocity $V$ due to the translation motion of the substrate and to move in the opposite direction due to the peeling front propagation, such that

$$
\overline{P_{0} P}(t)=\ell_{p}(t)-V t .
$$

The distance between the winding and peeling points can be written

$$
\begin{aligned}
|\mathbf{M P}|(t) & =\sqrt{\left(\mathbf{M P}_{\mathbf{0}}+\mathbf{P}_{\mathbf{0}} \mathbf{P}(t)\right)^{2}}, \\
& \simeq\left|\mathbf{M} \mathbf{P}_{\mathbf{0}}\right|+\overline{P_{0} P} \cos \theta_{0},
\end{aligned}
$$

where $\theta_{0}$ corresponds to the peeling angle when the peeling front is at location $P_{0}$. The approximation made in Eq. (3) is valid to a precision better than $0.4 \%$ in our experiments since in practice we always observe that $\left|\overline{P_{0} P}\right|<410^{-2}\left|\mathbf{M P}_{\mathbf{0}}\right|$. The distance $|\mathbf{M P}|(t)$ can also be related to the elongation $u(t)$ through the equation

$$
|\mathbf{M P}|(t)=\mathscr{L}(t)+u(t),
$$

where $\mathscr{L}(t)$ is the unstretched peeled tape length. $\mathscr{L}(t)$ is not a constant and varies according to the rate of tape creation at the peeling front and of tape disappearance at the winding point

$$
\mathscr{L}(t)=\mathscr{L}_{0}+\int_{0}^{t}\left(v_{p}(\tau)-R \omega \frac{\mathscr{L}(\tau)}{|\mathbf{M P}|(\tau)}\right) d \tau
$$

Here, the tape on the substrate is assumed to have been applied in an unstretched state while the tape wound on the cylinder is stretched. As already discussed in section 2, in our experiments, the stretch ratio $|\mathbf{M P}| / \mathscr{L}$ departs from 1 of typically $0.1 \%$ to $1.5 \%$ and the winding roller radius $R(t)$ slowly increases during the peeling. Practically, the velocity of the winding $\omega$ in the stationary stage is set to a constant value such that $\omega R(t) \frac{\mathscr{L}(t)}{|\mathbf{M P}|(t)}$ matches $V$ to a precision always better than $1.5 \%$ at the worst moments (of the less favorable experiments): the match is most of the time much better. We can therefore safely approximate (5) by

$$
\mathscr{L}(t) \simeq \mathscr{L}_{0}+\int_{0}^{t}\left(v_{p}(\tau)-V\right) d \tau=\mathscr{L}_{0}+\ell_{p}(t)-V t .
$$

This approximation actually amounts to neglect the drift during a stick-slip cycle of the peeling point position $\ell_{p}(t)-V t$ in the laboratory frame due to the mismatch between $\omega R(t) \frac{\mathscr{L}(t)}{|\mathbf{M P}|(t)}$ and $V$ with respect to its stick-slip oscillation amplitude: This approximation is truly relevant since the former is never larger than $2 \%$ of the latter in our experiments. 
From Eqs. (2-6), we obtain the following kinematical constraint for the peeled tape elongation

$$
u=u_{0}+\left(V t-\ell_{p}\right)\left(1-\cos \theta_{0}\right),
$$

where $u_{0}=\left|\mathbf{M P}_{\mathbf{0}}\right|-\mathscr{L}_{0}$ is the mean elongation during the peeling. In our geometry, the extension of Barquins et al.'s equation ${ }^{7}$ for any peeling angle is thus

$$
\frac{d u}{d t}=\left(V-v_{p}\right)\left(1-\cos \theta_{0}\right)
$$

If we assume a uniform tensile stress in the ribbon, we can further write

$$
F=\frac{E b e}{\mathscr{L}} u \simeq \frac{E b e}{L} u,
$$

(the approximation $\mathscr{L} \simeq L=\left|\mathbf{M P}_{\mathbf{0}}\right|$ being valid to a precision better than $1 \%$ and generally much better). From Eq. (1), we finally deduce the energy release rate

$$
G \simeq \frac{E e}{L}(1-\cos \theta(t))\left[u_{0}+\left(V t-\ell_{p}\right)\left(1-\cos \theta_{0}\right)\right],
$$

where $\theta(t)$ corresponds to the peeling angle at location $P(t)$.

In our geometry (see Fig. 1), we have

$$
\cos \theta(t)=\frac{\left|\mathbf{M P}_{\mathbf{0}}\right| \cos \theta_{0}+\overline{P_{0} P}}{\sqrt{\left|\mathbf{M P}_{\mathbf{0}}\right|^{2}+\left|\mathbf{P}_{\mathbf{0}} \mathbf{P}\right|^{2}+2 \mathbf{M} \mathbf{P}_{\mathbf{0}} \cdot \mathbf{P}_{\mathbf{0}} \mathbf{P}}} .
$$

Since $\left|\overline{P_{0} P}\right|<410^{-2}\left|\mathbf{M P}_{\mathbf{0}}\right|$, we can develop (11) to the first order in $\overline{P_{0} P} /\left|\mathbf{M P}_{\mathbf{0}}\right|=\left(\ell_{p}-V t\right) / L$ which leads to

$$
\cos \theta(t) \simeq \cos \theta_{0}+\frac{\ell_{p}-V t}{L}\left(1-\cos ^{2} \theta_{0}\right) .
$$

Finally injecting (12) into (10) and noting that $u_{0} / L=$ $\Gamma(V) / E e\left(1-\cos \theta_{0}\right)$ is typically of order of $10^{-3}$ for the studied adhesive tape* and therefore smaller than $\mid \ell_{p}-$ $V t \mid / L$, the energy release rate can be written to first order in $\left(\ell_{p}-V t\right) / L$

$$
G=\frac{E e}{L}\left(1-\cos \theta_{0}\right)\left[u_{0}+\left(V t-\ell_{p}\right)\left(1-\cos \theta_{0}\right)\right] .
$$

Its time derivative finally verifies

$$
\frac{\mathrm{d} G}{\mathrm{~d} t}=\frac{k_{\text {eff }}}{b}\left(V-v_{p}\right) .
$$

where $k_{\text {eff }}=\left(1-\cos \theta_{0}\right)^{2} E b e / L$ is an effective stiffness. We stress here that one of the factors $\left(1-\cos \theta_{0}\right)$ comes from the geometry dependence of the energy release rate,

\footnotetext{
${ }^{*}$ The fracture energy for Scotch ${ }^{\circledR} 600$ adhesive tapes is typically of the order of or less than $100 \mathrm{~J} \mathrm{~m}^{-212}$.
}

while the other one arises from the unstable peeling dynamics.

Finally, assuming that the quasistatic relation $G=\Gamma$ still holds instantaneously when peeling is not stationary, we obtain the following dynamical equation

$$
\left.\frac{d \Gamma}{d v_{p}}\right|_{v_{p}} \frac{d v_{p}}{d t}=\frac{k_{\mathrm{eff}}}{b}\left(V-v_{p}\right) .
$$

The stationary solution $v_{p}(t)=V$ of Eq. (15) becomes unstable as soon as the fracture energy $\Gamma\left(v_{p}\right)$ is a decreasing function. According to this simple approach, the instability onset should be the velocity $V_{a}$ at the end of the "slow" increasing branch of $\Gamma\left(v_{p}\right)$ where it reaches a local maximum (see Fig. 2 in $^{24}$ representing $\Gamma(V)$ for the Scotch ${ }^{\circledR} 600$ adhesive tapes). Therefore, one may ask if the effect of peeling angle on the stick-slip instability onset and amplitude could be related to a peeling angle dependance of the velocity $V_{a}(\theta)$ and more generally of the fracture energy $\Gamma\left(v_{p}, \theta\right)$. This dependance should however be strong enough to explain the reported change of an order of magnitude in the instability velocity range over the studied range of peeling angle. To our knowledge, there is no indication from the literature for such a strong angle dependence of the fracture energy. It has actually received only few experimental tests, which tend to rule out such peeling angle dependence ${ }^{25-27}$. The only evidences for an angular dependance of $\Gamma$ have been reported by Kaelble ${ }^{28}$, who relates it to a transition from cleavage to shearing loading of the adhesive at very small peeling velocities, and by Gent \& Hamed ${ }^{29}$, who relates it to the appearance of plastic deformations of the tape backing at large peeling angle. All these works however involve adhesives which are significantly different from ours. It would therefore be valuable to measure systematically the fracture energy dependence with peeling velocity and angle for the adhesive tape considered here.

On the contrary, previous experimental observations of the stick-slip instability of peeling ${ }^{30}$ have shown that increasing the stiffness of the loading system when peeling at $90^{\circ}$ leads to a reduction of the stick-slip unstable domain and may even suppress it entirely. A similar effect is known in the case of frictional stick-slip instability ${ }^{31}$. From that perspective, the reduction in the stick-slip velocity range with increasing peeling angle could be due to an increase in effective stiffness $k_{\text {eff }}$. Indeed, a strong increase of $k_{\text {eff }}$ by a factor of 200 is obtained when increasing the peeling angle from $\theta=30^{\circ}$ to $150^{\circ}$. Likewise, the lack of detectable effect of the peeled length on the unstable velocity range could simply be due to the corresponding weak variation in effective stiffness, limited to a factor of 5 in our experiment. From a theoretical point of view, the physical origin of the reduction of the unstable 
velocity range when the elastic stiffness of the loading becomes large is still unclear in the case of the adhesive tape peeling.

\subsection{Effect of the peeling angle on the in- stability limit cycles: an inertial ef- fect?}

In addition to the instability thresholds, it is also fundamental to carefully consider how the instability velocity fluctuations, once developed, are strongly dependent on the peeling angle.

For small angle, the stick-slip amplitude $\Delta v_{p}$ is nearly constant around the value $40 \mathrm{~m} \mathrm{~s}^{-1}$. These experiments actually correspond to the archetypal stick-slip relaxation dynamics, with the peeling being alternatively very slow during long stick phases and very fast during very short slip phases as illustrated in Fig. 4(a). Barquins and Maugis ${ }^{7,8}$ proposed that in this regime the stick-slip dynamics is such that the peeling explores quasistatically the "slow" stable velocity branch of $\Gamma\left(v_{p}\right)$ during the stick phase, i.e. $G(t)=\Gamma\left(v_{p}(t)\right)$, in alternance with infinitely short dynamical slip phases approaching the fast stable branch of $\Gamma\left(v_{p}\right)$ (see Fig. 2 in $^{24}$ representing $\Gamma(V)$ ). This theoretical framework leads to quantitative prediction of the stick period which have received several experimental validations ${ }^{7,8,24}$. It predicts in particular that the stickslip oscillation period decreases almost as $1 / V$ which we have checked to be valid for the data presented here for $\theta=30^{\circ}$. In the framework of this relaxation stick-slip dynamics, one would also expect very large values of $\Delta v_{p}$, independent of the average peeling velocity, exactly as observed in our experiments at $\theta=30^{\circ}$.

For large angles, in contrast, $\Delta v_{p}$ becomes dependant on the peeling velocity, converging towards $\Delta v_{p}=2 \mathrm{~V}$ (Fig. 4(c)), and the time evolution of $v_{p}$ during a stickslip cycle consists in nearly sinusoidal oscillations around $V$ (Fig. 4(b)). This behavior occurs at large effective stiffness $k_{\text {eff }} \propto(1-\cos \theta)^{2}$ which, according to Eq. (15), imposes a smaller and smaller quasistatic time scale for the velocity dynamics as $\theta$ increases. Whereas the quasistatic time scale gets smaller, some inertial effects may eventually become important: they could be associated to the propagation of elongation waves in the backing tape or to the changes in the tape bending close to the peeling fracture front. Before building a complete description of the interaction between the crack growth criterion at the peeling fracture front and the dynamics of the elastic deformations of the backing tape, one may try to take into account empirically the effective influence of these inertial effects on the quasistatic balance equation $G=\Gamma\left(v_{p}\right)$ by replacing it with a dynamical equation

$$
\mu \ddot{x}=G-\Gamma(\dot{x}),
$$

where $x$ is the position of the peeling front, so that $v_{p}=\dot{x}$, and $\mu$ represents a yet unknown effective inertial mass per unit length. Eq. (16) was originally proposed by Webb and Aifantis ${ }^{32}$ in order to describe oscillatory crack propagation in polymeric materials.

A stationary solution of Eq. (16) is $x_{s}=V t$ and $\dot{x}_{S}=V$. Introducing the fluctuations around the stationary solution $\delta x=x-x_{s}$ (hence, $\delta \dot{x}=\dot{x}-V$ ), Eq. (16) becomes

$$
\mu \delta \ddot{x}+\frac{k_{\mathrm{eff}}}{b} \delta x=\Gamma(V)-\Gamma(\dot{x}) .
$$

The left hand side of this equation corresponds to an harmonic oscillator with angular frequency $\omega=\sqrt{k_{\mathrm{eff}} / b \mu}$. As can be seen in Fig. 4(b), the experimental motion of the peeling front when the peeling angle is large is not very far from a sinusoidal oscillation. The corresponding experimental period of oscillations $T_{\mathrm{ss}}$ can be used to get an estimate of the effective mass per unit length $\mu$. For $\theta=150^{\circ}$ and $V=0.9 \mathrm{~m} \mathrm{~s}^{-1}$, we have for example $T_{\mathrm{ss}}=2.2 \pm 0.4 \mathrm{~ms}$ and $k_{\mathrm{eff}}=5.910^{3} \mathrm{~N} \mathrm{~m}^{-1}$, so that we would predict $\mu \simeq 0.038 \pm 0.013 \mathrm{~kg} \mathrm{~m}^{-1}$. This value is close to the mass of the peeled tape per unit width of the peeling front: $\alpha=0.024 \mathrm{~kg} \mathrm{~m}^{-1}$.

Furthermore, for the same experiment, we have measured stick-slip amplitudes of about $2 \mathrm{~mm}$. Taking therefore $\delta x=1 \mathrm{~mm}$, we find that $\frac{k_{\text {eff }}}{b} \delta x \simeq \pm 309 \mathrm{~J} \mathrm{~m}^{-2}$, hence an amplitude of about $600 \mathrm{~J} \mathrm{~m}^{-2}$. In the ideal stick-slip cycle described by Barquins et al., the variation of fracture energy corresponds to the two extreme values of the decreasing branch of $\Gamma(V)$. It was measured to be at most $100 \mathrm{~J} \mathrm{~m}^{-2}$ when peeling at $90^{\circ}$ from a roller for the $\operatorname{Scotch}^{\circledR} 600$ adhesive tapes ${ }^{24}$. Provided the order of magnitude of $\Gamma$ does not depend strongly on $\theta$, we can thus expect that fluctuations of elastic energy release rate are about 6 times larger than the fluctuations of fracture energy during a stick-slip cycle. This observation confirms that a strong discrepancy between $G$ and $\Gamma(\dot{x})$ may appear and shall be accounted for by a new physical term in the equations. If we do assume that $\Gamma(\dot{x})-\Gamma(V)$ can consequently be neglected at large peeling angle and that $\dot{x}=0$ when $u=0$, which is the case when the peeled tape has not been loaded yet, we obtain the following asymptotic solution of Eq. (17)

$$
\begin{aligned}
& x=V t-\frac{V}{\omega} \sin (\omega t), \\
& \dot{x}=V(1-\cos (\omega t)) .
\end{aligned}
$$

This asymptotic solution is consistent with the experimental velocity oscillation observed at large angle (Fig. 4(b)). 
It moreover predicts exactly $\Delta v_{p}=2 V$ for the instability amplitude which is the experimentally observed asymptotic limit.

This analysis shows that, at large peeling angle, when the effective stiffness of the peeled tape is large, the fracture energy seems to be eventually not important in the determination of the fracture velocity limit cycles, although it surely remains important to make the peeling unstable, i.e. to trigger the instability. For smaller peeling angles, we expect the unstable peeling dynamics to be due to a combination of inertial effects, geometry-dependent stiffness and fracture energy decreasing with fracture velocity. The fact that geometry has a determinant role on the dynamical instability of adhesive peeling, which is stronger than expected from the simple geometry dependence of the energy release rate, is intrinsically connected to the action of an effective inertial mass of the crack whose physical origin is still to be uncovered.

\section{Acknowledgments}

We thank A. Aubertin, L. Auffray, C. Borget and R. Pidoux for their help in the conception of the experiment, M. Ciccotti, C. Creton, and J.-P. Hulin for fruitful scientific discussions and F. Moisy, P. Holdsworth and T. Dauxois for a careful reading of the manuscript. This work has been supported by the French ANR through Grant "StickSlip" No. 12-BS09-014. The peeling experiments was funded by the University Paris-Sud.

\section{References}

[1] L.B. Freund, Dynamic fracture mechanics, Cambridge University Press, London, 1990.

[2] B. Lawn, Fracture of brittle solids, Cambridge University Press, 1995.

[3] J. Fineberg and M. Marder, Physics Reports, 1999, 313(1-2):1.

[4] K. Tsunoda, J.J.C. Busfield,C.K.L. Davies and A.G. Thomas, J. Mater. Sci., 2000, 35(20):5187.

[5] J.L. Gardon, J. Appl. Polym. Sci., 1963, 7, 625-641.

[6] D.W. Aubrey, G.N. Welding and T. Wong, J. Appl. Polym. Sci., 1969, 13, 2193-2207.

[7] M. Barquins, B. Khandani and D. Maugis, C. R. Acad. Sci. serie II,1986, 303, 1517-1519.
[8] D. Maugis and M. Barquins, Adhesion 12, edited by K. W. Allen, Elsevier ASP, London, 1988, pp. 205222 .

[9] A.N. Gent and R.P. Petrich, Proc. R. Soc. London, Ser. A, 1969, 310, 433-448.

[10] D.W. Aubrey and M. Sherriff, J. Polym. Sci.,1980, 18, 2597-2606.

[11] C. Derail, A. Allal, G. Marin and P. Tordjeman, J. Adhesion, 1997, 61, 123-157; 1998, 68, 203-228.

[12] M. Barquins and M. Ciccotti, Int. J. Adhes. Adhes., 1997, 17, 65-68.

[13] D. Maugis, J. Mater. Sci., 1985 20, 3041-3073.

[14] P.-G. de Gennes, Langmuir,1996, 12, 4497-4500.

[15] A.N. Gent, Langmuir, 1996, 12, 4492-4496.

[16] E. Barthel and C. Fretigny, J. Phys. D Appl. Phys., 2009, 42, 19.

[17] H. Tabuteau, S. Mora, M. Ciccotti, C.-Y. Hui and C. Ligoure, Soft Matter, 2011, 7, 9474-9483.

[18] R. De and G. Ananthakrishna, Phys. Rev. E, 2004, 70, 046223 .

[19] M. Ciccotti, B. Giorgini and M. Barquins, Int. J. Adhes. Adhes., 1998, 18, 35.

[20] R. De and G. Ananthakrishna, Phys. Rev. Lett., 2006, 97, 165503.

[21] M. Ciccotti, B. Giorgini, D. Vallet and M. Barquins, Int. J. Adhes. Adhes., 2004, 24, 143-151.

[22] P.-P. Cortet, M. Ciccotti and L. Vanel, J. Stat. Mech., 2007, P03005.

[23] P.-P. Cortet, M.-J. Dalbe, C. Guerra, C. Cohen, M. Ciccotti, S. Santucci and L. Vanel, Phys. Rev. E, 2013, 87, 022601.

[24] M.-J. Dalbe, S. Santucci, P.-P. Cortet and L. Vanel, Soft Matter, 2014, 10, 132.

[25] K. Kendall, J. Phys. D: Appl. Phys., 1975, 8, 14491452.

[26] D.H. Kaelble, Trans. Soc. Rheol. 3, 161 (1959).

[27] D. Maugis, M. Barquins, J. Phys. D: Appl. Phys. 11, 1989 (1978).

[28] D.H. Kaelble, Trans. Soc. Rheol. 4, 45 (1960). 
[29] A.N. Gent, G.R. Hamed, J. Appl. Pol. Sci. 21, 2817 (1977).

[30] Y. Yamazaki and A. Toda, Physica D, 2006, 214, 120-131.

[31] T. Baumberger and C. Caroli, Adv. Phys., 2006, $\mathbf{5 5}(3), 279$.

[32] T. Webb and E. Aifantis, Int. J. Solids Struct., 1995, 32, 2725-2743. 